\title{
Search, propagation, and learning in sequencing and scheduling problems
}

\author{
Mohamed Siala $^{1,2}$
}

Published online: 15 September 2015

(C) Springer Science+Business Media New York 2015

\begin{abstract}
Sequencing and scheduling involve the organization in time of operations subject to capacity and resource constraints. We propose in this dissertation several improvements to the constraint satisfaction and combinatorial optimization methods for solving these problems. These contributions concern three different aspects: how to choose the next node to explore (search)? how much, and how efficiently, one can reduce the search space (propagation)? and what can be learnt from previous failures (learning)?
\end{abstract}

Our contributions start with an empirical study of search heuristics for the well known carsequencing problem. This evaluation characterizes the key aspects of a good heuristic and shows that the search strategy is as important as the propagation aspect in this problem. Second, we carefully investigate the propagation aspect in a class of sequencing problems. In particular, we propose an algorithm for filtering a type of sequence constraints which worst case time complexity is lower than the best known upper bound, and indeed optimal. Third, we investigate the impact of clause learning for solving the car-sequencing problem. In particular, we propose reduced explanations for the new filtering. The experimental evaluation shows compelling evidence supporting the importance of clause learning for solving efficiently this problem. Next, we revisit the general approach of lazy generation for the Boolean variables encoding the domains. Our proposition avoids a classical redundancy issue without computational overhead. Finally, we investigate conflict analysis algorithms for solving disjunctive scheduling problems. In particular, we introduce a novel learning procedure tailored to this family of problems. The new conflict analysis differs from conventional methods by learning clauses whose size are not function of the scheduling horizon. Our comprehensive experimental study with traditional academic benchmarks demonstrates the impact of the novel learning

Mohamed Siala

sia.mohamed@gmail.com

1 CNRS, LAAS, 7 Avenue du Colonel Roche, F-31400 Toulouse, France

2 Univ de Toulouse, INSA, LAAS, F-31400 Toulouse, France 
scheme that we propose. In particular, we find new lower bounds for a well known scheduling benchmark.

School: Université Fédérale Toulouse Midi-Pyrénées

\section{Supervisors:}

Emmanuel Hebrard

Christian Artigues

Graduated: Wednesday, May 13, 2015

Link to full text: http://www.a4cp.org/sites/default/files/mohamed_siala_-_search_ propagation_and_learning_in_sequencing_and_scheduling_problems.pdf

Notes:

"Honorable mention" award for the paper "An Optimal Arc Consistency Algorithm for a Chain of Atmost Constraints with Cardinality", 18th International Conference on Principles and Practice of Constraint Programming, CP 2012 